PREPARED FOR THE U.S. DEPARTMENT OF ENERGY, UNDER CONTRACT DE-AC02-76CH03073

PPPL-3588

PPPL-3588

UC-70

Two-dimensional Simulations of Correlation

Reflectometry in Fusion Plasmas

by

E.J. Valeo, G.J. Kramer, and R. Nazikian

July 2001

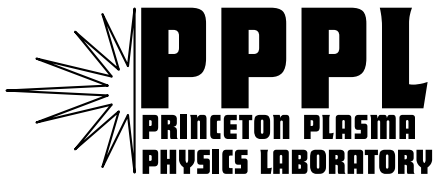

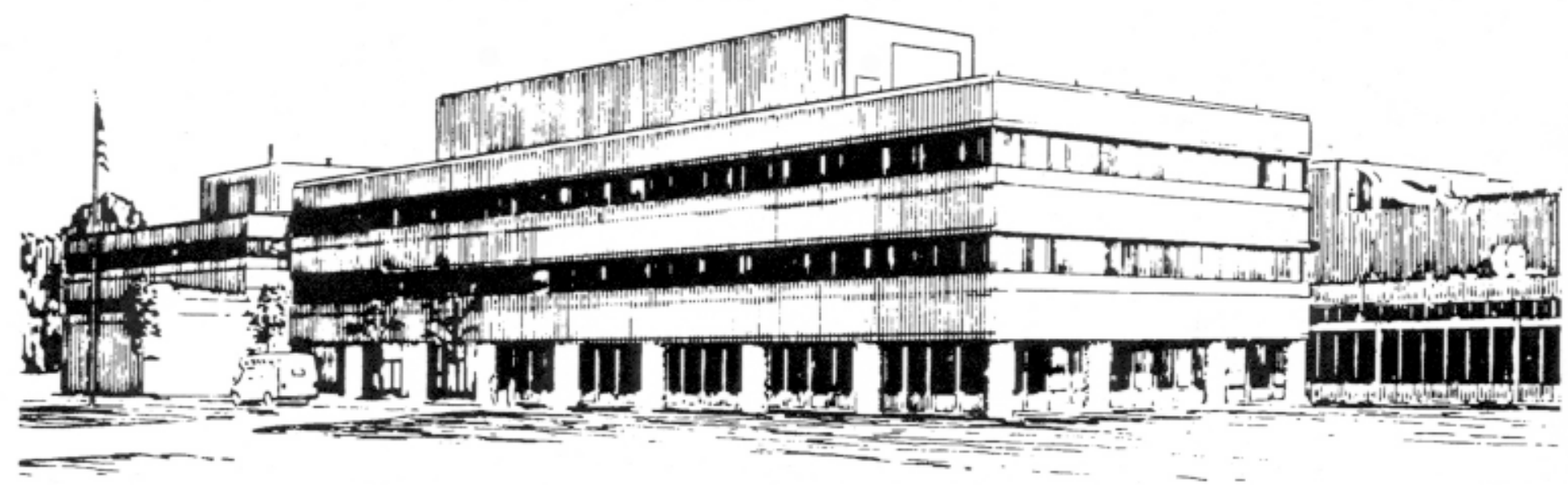

PRINCETON PLASMA PHYSICS LABORATORY PRINCETON UNIVERSITY, PRINCETON, NEW JERSEY 


\section{PPPL Reports Disclaimer}

This report was prepared as an account of work sponsored by an agency of the United States Government. Neither the United States Government nor any agency thereof, nor any of their employees, makes any warranty, express or implied, or assumes any legal liability or responsibility for the accuracy, completeness, or usefulness of any information, apparatus, product, or process disclosed, or represents that its use would not infringe privately owned rights. Reference herein to any specific commercial product, process, or service by trade name, trademark, manufacturer, or otherwise, does not necessarily constitute or imply its endorsement, recommendation, or favoring by the United States Government or any agency thereof. The views and opinions of authors expressed herein do not necessarily state or reflect those of the United States Government or any agency thereof.

\section{Availability}

This report is posted on the U.S. Department of Energy's Princeton Plasma Physics Laboratory Publications and Reports web site in Calendar Year 2001. The home page for PPPL Reports and Publications is: http://www.pppl.gov/pub_report/

DOE and DOE Contractors can obtain copies of this report from:

U.S. Department of Energy

Office of Scientific and Technical Information

DOE Technical Information Services (DTIS)

P.O. Box 62

Oak Ridge, TN 37831

Telephone: (865) 576-8401

Fax: (865) 576-5728

Email: reports@adonis.osti.gov

This report is available to the general public from:

National Technical Information Service

U.S. Department of Commerce

5285 Port Royal Road

Springfield, VA 22161

Telephone: 1-800-553-6847 or

(703) 605-6000

Fax: (703) 321-8547

Internet: http://www.ntis.gov/ordering.htm 


\title{
Two-dimensional Simulations of correlation Reflectometry in fusion Plasmas
}

\author{
E. J. Valeo, G. J. Kramer, and R. Nazikian \\ Princeton University \\ Plasma Physics Laboratory \\ Princeton, NJ 08543
}

July 3,2001

\begin{abstract}
A two-dimensional wave propagation code, developed specifically to simulate correlation reflectometry in large scale fusion plasmas is described. The code makes use of separate computational methods in the vacuum, underdense and reflection regions of the plasma in order to obtain the high computational efficiency necessary for correlation analysis. Simulations of TFTR plasma with internal transport barriers are presented and compared with one-dimensional full-wave simulations. It is shown that the two-dimensional simulations are remarkably similar to the results of the one-dimensional full-wave analysis for a wide range of turbulent correlation lengths. Implications for the interpretation of correlation reflectometer measurements in fusion plasma are discussed.
\end{abstract}

*email: valeo@pppl.gov 


\section{Introduction}

Reflectometry[1, 2] is routinely used to infer turbulent fluctuation levels and correlation lengths in both small-scale laboratory plasma[3, 4] and large-scale fusion research devices[5]. The basic technique is to launch an electromagnetic wave with frequency chosen so that there exists a reflection layer in the plasma. The time-dependent reflected signal is collected either by the transmitting antenna, or by a nearby receiving antenna. The time-dependent product of the reflected signal and a local oscillator is accumulated.

The measurements are of two types: the coherent reflected signal strength measured at the transmitting antenna and the cross correlation between signals at a reference frequency, $\omega_{0}$ and a number of secondary frequencies $\omega_{1}$, chosen so that the range of reflection points encompasses the turbulent correlation length. The cross-correlation signal monotonically decays with increasing separation between reflection points.

Computationally, various models have been applied to simulate the signal expected for models of turbulent fluctuations imposed on an otherwise smooth plasma profile. One-dimensional models, either in the geometrical optics approximation[6] or solutions of the full-wave equation[7] have been routinely used to infer the relation between measured correlation functions and the properties of the turbulent fluctuations. Mazzucato[8] extended the one-dimensional analysis by considering scattering from two-dimensional turbulence in a planar plasma profile. Recently, Lin et al.[9] have solved the two-dimensional full-wave equation for a single scattering realization in realistic geometry, appropriate to Alcator CMOD. However, to date, two-dimensional full wave analysis has not been applied to computation of correlation reflectometry.

In this paper we describe a two-dimensional wave propagation code which has been constructed in order to help interpret the experimental signals from large scale tokamak experiments. To facilitate quantitative comparison between computational and experimental results, the model includes a realistic antenna radiation pattern and the capability to input experimentally inferred two-dimensional profiles[10, 11] of density, electron temperature and magnetic field strength. We find that the convergence of the coherent and cross-correlation signals is quite slow with increasing ensemble size - typically several hundred realizations are required to obtain a meaningful result. An efficient 
computational algorithm is therefore a prerequisite if a model is to be a useful part of the data analysis process. The algorithm presented here is sufficiently fast (requiring $30 \mathrm{CPU}$ seconds for computation of a single realization in a large tokamak) to make this practical. Efficiency is obtained by several means. First, the computational domain is divided into regions as shown in Figure (1). The full-wave equation is solved only where necessary (near the reflection layer). An implicit algorithm is used to speed approach to steady state. Elsewhere, computationally less demanding models suffice: The paraxial approximation is used in the underdense plasma. The wave field is projected through vacuum using the free-space Green's function.

The model is described in Section II. Results are presented in Section III, in which a comparison of one- and two-dimensional results for experimental profiles in a TFTR discharge is shown. It is found that the results of the two-dimensional correlation analysis bear a remarkable similarity to those obtained from the one-dimensional full wave simulations over a wide range of turbulent scale lengths. Implications of this result for the interpretation of correlation reflectometry and future directions are presented in Section IV.

\section{Computational Model}

The propagation of the electric field amplitude $E(\mathbf{x}, t)$ is described by the wave equation

$$
2 i \omega \frac{\partial E}{\partial t}+\mathcal{L} E=0
$$

with

$$
\mathcal{L} \equiv c^{2} \nabla^{2}+\omega^{2} \epsilon .
$$

where we have made the assumption of quasi-monochromaticity at frequency $\omega$. Here $E(\mathbf{x}, t)$ is a complex field amplitude, in terms of which the full time-space variation is given as

$$
\mathcal{E}(\mathbf{x}, t)=\Re[\exp (-i \omega t) E(\mathbf{x}, t)]
$$

where $\Re$ designates the real part. The time variation of $E(\mathbf{x}, t)$ is assumed slow compared to that of the phase factor. The plasma dielectric $\epsilon$ is modeled by the magnetized cold plasma X or O mode dielectric[12] with electron thermal corrections added[13, 14]. For

large tokamaks, thermal effects result in significant shifts in the reflection point relative to the cold plasma result. 
Since the wave transit time $\left(\sim L_{n} / v_{g r} \sim 100 \mathrm{~cm} / c \sim 3 \cdot 10^{-9} \mathrm{sec}\right)$ is short compared to the time over which the turbulent fluctations vary $\left(\sim 10^{-5} \mathrm{sec}\right)$, a set of steady solutions is computed for a given macrosopic profile and microwave parameters. The relative amplitude $\delta n_{\mathbf{k} s}$ of the microscopic fluctuations at each wavevector $\mathbf{k}$ for simulation $s$ is assigned in terms of an assumed fluctuation spectrum $I_{\mathbf{k}}$, specifically $\left|\delta n_{\mathbf{k} s}\right| \propto I_{\mathbf{k}}^{1 / 2}$. The phases of the fluctuations are chosen randomly. Thus, for simulations $s$ and $s^{\prime}$, and wavevectors $\mathbf{k}$ and $\mathbf{k}^{\prime}$, the fluctuation correlation satisfies

$$
\left\langle\delta n_{\mathbf{k} s}^{*} \delta n_{\mathbf{k}^{\prime} s^{\prime}}\right\rangle=\delta_{\mathbf{k k}^{\prime}} \delta_{s s^{\prime}} I_{\mathbf{k}}
$$

The inverse Fourier transform of $\delta n_{\mathbf{k}}$ yields the relative fluctuation amplitude $\delta n(\mathbf{x}) / n_{0}(\mathbf{x})$ where $n_{0}(\mathbf{x})$ is the smoothly varying equilibrium density profile. The total density

$$
n(\mathbf{x})=n_{0}(\mathbf{x})[1+\delta n(\mathbf{x})]
$$

For comparison with experimental data the spectral intensity is parameterized by an amplitude $I_{0}$, a mean wavevector $\mathbf{k}_{m}$ and a twodimensional width $\Delta \mathbf{k}$. The specific form chosen for the simulation results presented below is

$$
I_{\mathbf{k}}=I_{0} \exp -\left\{\left[\frac{\left(\mathbf{k}-\mathbf{k}_{m}\right) \cdot \hat{\mathbf{R}}}{\boldsymbol{\Delta} \mathbf{k} \cdot \hat{\mathbf{R}}}\right]^{2}+\left[\frac{\left(\mathbf{k}-\mathbf{k}_{m}\right) \cdot \hat{\mathbf{Z}}}{\Delta \mathbf{k} \cdot \hat{\mathbf{Z}}}\right]^{2}\right\}
$$

where $\hat{\mathbf{R}}$ (resp. $\hat{\mathbf{Z}}$ ) are unit vectors in the radial (resp. vertical) directions in the poloidal plane. Many (usually several hundred) runs are made for a given choice $\left(I_{0}, \mathbf{k}_{m}, \Delta \mathbf{k}\right)$. An average over the solution ensemble is identified with the experimentally obtained time series data.

A major challenge is that the computational domain - a poloidal cross section - measures many wavelengths in both dimensions. The wavelength of the probing radiation is set by the parameters at the desired reflecting layer. In large scale tokamaks such as JT60 and TFTR, the wavelength is 2 to $3 \mathrm{~mm}$. The distance between the antenna and reflecting layer is typically 2 meters, of which approximately $50-100 \mathrm{~cm}$ is in plasma, with the remainder in vacuum. Propagation in vacuum is most efficiently handled by making use of the free-space Green's function to project the wave field between the antenna and plasma boundary. Considering, then, only the plasma region for direct 
numerical solution, the radial extent of the computational domain, $L_{R} \sim 400 \lambda$. Even for a well-collimated incident beam, curvature of the reflecting layer and turbulent scattering typically lead to a finite cone angle of order $\pm 30^{\circ}$ for the reflected radiation. Thus the vertical extent $L_{Z}$ is also of order $400 \lambda$.

An explicit solution of the wave equation requires a timestep which satisfies the Courant stability condition. Assume discretization on a grid of cell size $\Delta d=\lambda / n_{d}$ with $n_{d}$ points per wavelength in the $d=$ $\{R, Z\}$ direction. Then, $\Delta t \leq \min (\Delta R, \Delta Z) /(2 c)$, with $c$ the speed of light. Solving for a time of order the crossing time $\tau_{c r} \sim 2 L_{R} / c$, requires $N_{t}=\max \left\{N_{R}, N_{Z}\right\}$ time steps with $N_{d}=n_{d} L_{d} / \lambda$. The CPU time per time step $\tau_{\text {step }}=C N_{R} N_{Z}$, with $C \sim 5 \cdot 10^{-6}$ sec on a fast RISC workstation. Typically $n_{R}=20, n_{Z}=2$, so that $N_{t} \sim 10^{4}$ and $N_{R} N_{Z} \sim 10^{7}$. A single such run takes of order 100 CPU hours. Hundreds of runs are required to achieve statistically significant results for a given set of parameters, making an explicit solution impractical.

For these reasons we use an implicit algorithm, which is stable for arbitrarily large time step, limited only by accuracy considerations. We write

$$
\left(\frac{2 i \omega}{\Delta t}+\frac{1}{2} \mathcal{L}\right) E^{n+1}=S^{n}
$$

for $E^{n+1} \equiv E[(n+1) \Delta t]$. Here the source

$$
S^{n}=\left(\frac{2 i \omega}{\Delta t}+\frac{1}{2} \mathcal{L}\right) E^{n},
$$

involves quantities at only the $n^{\text {th }}$ time level. Inversion of the Laplacian on the left hand side then becomes the limiting step. By making use of the observation that, experimentally, the wavevectors are aligned principally along one of the (cartesian) coordinate directions $(R)$, an efficient iterative solution can be employed - the line Jacobi method[15]. Introducing iteration index $m$, the Laplacian $(\mathcal{L}=$ $\left.\mathcal{L}_{R}+\mathcal{L}_{Z}\right)$ is solved directly in $R$ and iteratively in $Z$ :

$$
\left(\frac{2 i \omega}{\Delta t}+\frac{1}{2} \mathcal{L}_{R}\right) E^{n+1, m}=-\frac{1}{2} \mathcal{L}_{Z} E^{n+1, m-1}+S^{n} .
$$

Taking $m=5$ is sufficient, given the rapid convergence.

The solution time for realistic large tokamak profiles on four Compaq 750Mhz Alpha 21264 processors (with domain decomposition into radial strips, and with essentially perfect scaling with processor number) is of order 10 minutes - marginally acceptable for required throughput. 
Because $|c \nabla \epsilon / \omega \epsilon|<<1$ away from the reflection layer, further efficiency is obtained there through the use of the paraxial approximation. Defining the radial wavevector $k_{R}(R) \equiv \sqrt{\epsilon\left(R, Z_{0}\right)}$ and phase $\phi \equiv \int^{R} d R k_{R}$ along the centroid, $Z=Z_{0}$, of the incident wave packet and decomposing the field into incoming (I) and reflected (R) components,

$$
E(\mathbf{x}, t)=E_{P I} \exp (-i \phi)+E_{P R} \exp (i \phi)
$$

we have, away from the reflection point,

$$
\pm 2 i \frac{\partial}{\partial R} k_{R}^{1 / 2} E_{P}+\mathcal{L}_{Z} k_{R}^{1 / 2} E_{P}+\left[\epsilon(R, Z)-\epsilon\left(R, Z_{0}\right)\right] k_{R}^{1 / 2} E_{P}=0,
$$

with the $+($ resp. -$)$ sign taken for $E_{P I}\left(\right.$ resp. $\left.E_{P R}\right)$ and where the time variation is ignored as slow compared to the wave transit time across the simulation domain. Again, the solution in $R$ is done implicitly, requiring an inversion of $\mathcal{L}_{Z}$. This is done directly through Gaussian elimination.

In summary, as shown in Figure (2), the computation proceeds as follows:

1. Specify the (complex) incident amplitude at the antenna plane.

2. Project the incident amplitude onto the plasma boundary using the free space Green's function.

3. Solve the paraxial equation for $E_{P I}$ up to a surface $R=R_{F W}$ within a few wavelengths of the reflection point.

4. From $R_{F W}$ inward solve the full-wave equation, Eq. (1), implicitly for $E$, with $E_{P I}$ as the incoming wave amplitude. Advance the solution until steady state is reached.

5. Take the outgoing component $E_{R}$ of $E$ as an initial condition for the reflected paraxial field

$$
E_{P R}\left(R_{F W}, Z\right)=E_{R}\left(R_{F W}, Z\right) .
$$

Apply Step 3, then Step 2 in the outgoing direction to compute the amplitude of the reflected field at the receiver plane.

The method is efficient and accurate. A single realization for a system size of $360 \times 240 \lambda^{2}$ takes $30 \mathrm{CPU}$ seconds to solve on a single alpha processor. 


\section{$3 \quad$ Results}

A principal result of the computations is the value of both the coherent reflected signal, $g(\omega)$, and the cross correlation $r\left(\omega_{0}, \omega_{1}\right)$. These are constructed from the numerical results as follows: For probe frequency $\omega$ let $E_{A I}(\omega, Z)$ and $E_{A R}(\omega, Z)$ designate the complex incident and reflected field amplitudes at $R=R_{A}$, with $R_{A}$ the radius of the antenna plane (Figure 2, right hand side). For any function $\Phi\left(R=R_{A}, Z\right)$, define the projection

$$
M(\omega) \equiv \int d Z E_{A R}(\omega, Z) E_{A I}^{*}(\omega, Z),
$$

Let \langle\rangle denote an average over the run ensemble. Then

$$
g(\omega) \equiv \frac{\langle M(\omega)\rangle}{\sqrt{\left\langle|M(\omega)|^{2}\right\rangle}},
$$

and

$$
r\left(\omega_{0}, \omega_{1}\right) \equiv \frac{\left\langle M\left(\omega_{0}\right) M\left(\omega_{1}\right)\right\rangle}{\sqrt{\left\langle\left|M\left(\omega_{0}\right)\right|^{2}\right\rangle\left\langle\left|M\left(\omega_{1}\right)\right|^{2}\right\rangle}} .
$$

Results for $g$ and $r$ from two series of simulations are presented in Figure 3, together with their values as calculated from a 1-D full wave simulation. Both were done for a typical TFTR plasma (shot \#65601, at time $2.5 \mathrm{sec}$ ). The main plasma parameters are: major radius: $2.93 \mathrm{~m}$, minor radius: $1.06 \mathrm{~m}$, magnetic field: $4.0 \mathrm{~T}$, central density: $1.0 \cdot 10^{20} \mathrm{~m}^{-3}$, and central electron temperature: $7.0 \mathrm{keV}$. Xmode waves were launched horizontally into the plasma midplane at 11 frequencies between 121 and $137 \mathrm{GHz}$ (vacuum wavelengths between 0.248 and $0.219 \mathrm{~cm}$ ) from an antenna of focal length $135 \mathrm{~cm}$ located at $R=400 \mathrm{~cm}, Z=0$.

One series (left hand side of Figure 3 ) was performed for a turbulent radial correlation length $\lambda_{c R}=0.2 \mathrm{~cm}$, the other for $\lambda_{c R}=$ $2.0 \mathrm{~cm}$. The poloidal correlation length $\lambda_{c Z}=0.4 \mathrm{~cm}$. The short poloidal correlation length was chosen so as to maximize the difference expected between two-dimensional and one-dimensional simulations. The volume-averaged relative fluctuation level, $f=\int_{V} d^{2} x(\delta n / n)^{2} / V=$ $2.5 \cdot 10^{-5}$. These parameters were achieved by choosing, in Eq. (6), $\Delta \mathbf{k} \cdot \hat{\mathbf{R}}=10 \mathrm{~cm}^{-1}$ and $1 \mathrm{~cm}^{-1}, \Delta \mathbf{k} \cdot \hat{\mathbf{Z}}=5 \mathrm{~cm}^{-1}, \mathbf{k}_{m}=0$, and $I_{0}=$ $4 \pi(\Delta \mathbf{k} \cdot \hat{\mathbf{R}})(\Delta \mathbf{k} \cdot \hat{\mathbf{Z}}) f$.

It can be seen that the calculated 2-D cross correlation agrees remarkably well with the $1 \mathrm{D}$ calculations[7] over this wide variation of 
radial correlation lengths. The fact that the coherent reflected signal is systematically higher in the $2 \mathrm{D}$ calculations can be explained by the scattering of waves out of the receiver aperture. Nevertheless, it is remarkable that the one- and two-dimensional coherent refleciton coefficients bear such a strong similarity, given the very short poloidal correlation lengths used in these simulations.

Shown in Figure 4 is the variation in $|g|$ with number of runs in the ensemble. Because of the slow rate of convergence, five hundred simulations were performed at each frequency. Thus, each point in Figure 3 represents an ensemble average of 500 separate runs.

A measure of the resolution expected from correlation reflectometry is shown in Figure 5 where the full-width half maximum of $|r|$ is plotted vs. that of the radial density correlation length both from the 2-D simulations (squares) as well as from a series of 1-D simulations (solid line). Again, one- and two-dimensional results are nearly identical and indicate a resolution limit less than $1 \mathrm{~cm}$.

\section{Discussion}

The surprising result of this analysis is that the correlation and coherent reflection level is essentially identical to that obtained with a 1-D full-wave analysis despite the fact that the transverse wavenumber of the perturbations is of the order of the wavelength of the probing beam. To understand this result in greater detail, many more simulations are certainly required. However, in a recent work, [9] it was pointed out that the curvature of the reflecting layer can have a significant effect to broaden the spectral response of the receiver for high transverse wavenumbers. Similar results have also been obtained using a 2-D full-wave analysis on the JT-60U tokamak[16], suggesting that the close correspondence of 2-D and 1-D simulations observed in our analysis may also hold for other large scale devices. However, the same analysis outlined in this paper needs to be repeated on a case by case basis to confirm the similarity of $2-\mathrm{D}$ and $1-\mathrm{D}$ simulations. Indications that the 2-D and 1-D simulations can also diverge significantly in their predictions is shown in the case of X-mode to O-mode correlation reflectometry performed on a laboratory scale facility [7]. In that case, the different radiation patterns at the reflecting layer for very different wave frequencies can lead to a degradation of the correlation coefficient at low fluctuation levels which is not apparent 
in the 1-D analysis.

These results were computed only for microwave beams launched normal to the surface of reflection. An important issue for future investigation is how sensitive the correspondense of the $1-\mathrm{D}$ and $2-\mathrm{D}$ simulations of the radial correlation are to small angles of misalignment of the waves incident on the reflecting layer or for a receiver slightly misaligned to the direction of specular reflection. Such systematic studies of correlation reflectometry in large scale facilities are now computationally tractable with the advent of efficient full-wave algorithm as discussed in this study.

\section{Conclusion}

A two-dimensional simulation program has been developed specifically to model reflectometry measurements in large tokamaks. By implementing algorithms tailored to the vacuum, underdense plasma and reflection layer regions, the high efficiency required for statistical studies has been achieved.

Results have been presented for a relatively large and relatively short radial correlation length of model density fluctuations. These results pertain to waves injected at normal incidence to the surface of reflection. Future studies will include an investigation of the degree to which the correspondence of one-dimensional and two-dimensional simulations breaks down with oblique angles of incidence.

The authors thank Doug McCune for help with the importation of TRANSP generated TFTR profiles.

This work is funded by DOE Contract No. DE-AC02-76CH03073. 


\section{References}

[1] Nazikian R, Kramer G J and Valeo E 2001 Phys. Plasma 81840

[2] Mazzucato E and Nazikian R 1991 Plasma Phys. Control. Fusion 33 L261

[3] Gilmore M, Peebles W A, and Nguyen X V 2000 Plasma Phys. Control. Fusion 42655

[4] Gilmore M, Peebles W A, and Nguyen X V 2000 Rev. Sci. Instrum. 72293

[5] Nazikian R, et al. 1998 IAEA-CN-69/PDP/03, paper presented at 17th IAEA Conf. on Fusion Energy, Yokohama

[6] Nazikian R and Mazzucato E 1995 Rev. Sci. Instrum. 66392

[7] Kramer G J, Nazikian R and Valeo E 2001 submitted to Plasma Phys. Control. Fusion

[8] Mazzucato E 1998 Rev. Sci. Instrum. 691691

[9] Lin Y, Nazikian R, Irby J H and Marmar E S 2001 Plasma Phys. Control. Fusion 43

[10] Hawryluk R J 1980 "An Empirical Approach to Tokamak Transport", in Physics of Plasmas Close to Thermonuclear Conditions ed. by B. Coppi, et al. (Brussels: CEC) 119

[11] Ongena J, Evrard M, McCune D 1998 Proceedings of the Third Carolus Magnus Summer School on Plasma Physics (Belgium: Spa), as published in Transactions of Fusion Technology, 33 No. $2 \mathrm{~T} 181$

[12] Stix T H 1992 "Waves in Plasmas" (New York: American Institute of Physics) section 2-7

[13] Mazzucato E 1992 Phys. Fluids B4 3460

[14] Bindslev H 1993 Plasma Phys. Control. Fusion 351093

[15] Vu H X 1998 Journ. Comp. Phys. 144257

[16] R. Nazikian, in preparation. 


\section{Figure Captions}

FIGURE 1. Electron density profile $N_{e}(R, Z)$ used for reflectometry simulations. The profile is reconstructed from TFTR Enhanced Reverse Shear shot \#65601 at time 2.5 sec. The rectangular domains labeled "FULL WAVE" (resp. "PARAXIAL APPROXIMATION") are the regions in which Eq. (1) (resp. Eq. (10)) are solved. The solutions are matched on the dotted strip $R=319 \mathrm{~cm},-13 \mathrm{~cm} \leq Z \leq 13 \mathrm{~cm}$.

FIGURE 2. Counterclockwise, from upper right: (1.) Real (blue) and Imaginary (red) components of the prescribed incident field amplitude $E_{A I}(Z)$ at the antenna plane, $R=400 \mathrm{~cm}$. Pseudocolor plots of: (2.) The intensity of the incident component $\left|E_{P I}(R, Z)\right|^{2}$ in the paraxial domain (3.) $|E(R, Z)|^{2}$ in the full-wave region. (4.) The intensity of the reflected component $\left|E_{P R}(R, Z)\right|^{2}$ in the paraxial domain. (5.) The final subplot shows the outgoing field amplitude $E_{A R}(Z)$ at the antenna plane, as in subplot (1). The pseudocolor plots extend over subsets of the respective computational domains in which the field strengths are non-negligible. The computational domain for the paraxial solution extends from $319 \mathrm{~cm} \leq R \leq 400 \mathrm{~cm}$, $-40 \mathrm{~cm} \leq Z \leq 40 \mathrm{~cm}$. The computational domain for the full-wave solution extends from $313 \mathrm{~cm} \leq R \leq 319 \mathrm{~cm},-13 \mathrm{~cm} \leq Z \leq 13 \mathrm{~cm}$.

FIGURE 3. Top: Magnitude of the cross-correlation coefficient $\left|r\left(\omega_{0}, \omega_{1}\right)\right|$ vs. the separation between reflection points. Bottom: The magnitude of coherent signal $|g(\omega)|$ vs. location of the reflection point at the plasma midplane relative to that for $f=128 \mathrm{GHz}$. Left hand plots are for a radial correlation length $\lambda_{c R}=0.2 \mathrm{~cm}$. Right hand plots are for $\lambda_{c R}=2.0 \mathrm{~cm}$. Other parameters: Volume averaged relative fluctuation level $=2.5 \cdot 10^{-5}$, poloidal correlation length $\lambda_{c Z}=0.4 \mathrm{~cm}$.

FIGURE 4. Measurement of the ensemble averaged coherent reflected signal $g$, Eq. (13), at a probe frequency of $128 \mathrm{GHz}$ vs. the number of simulations included in the ensemble. 
FIGURE 5. Measured value of the full-width half-maximum of $\left|r\left(\omega_{0}, \omega_{1}\right)\right|$ vs. that of the density correlation function from full-wave 1-D simulations (solid line) and 2-D simulations (squares). 


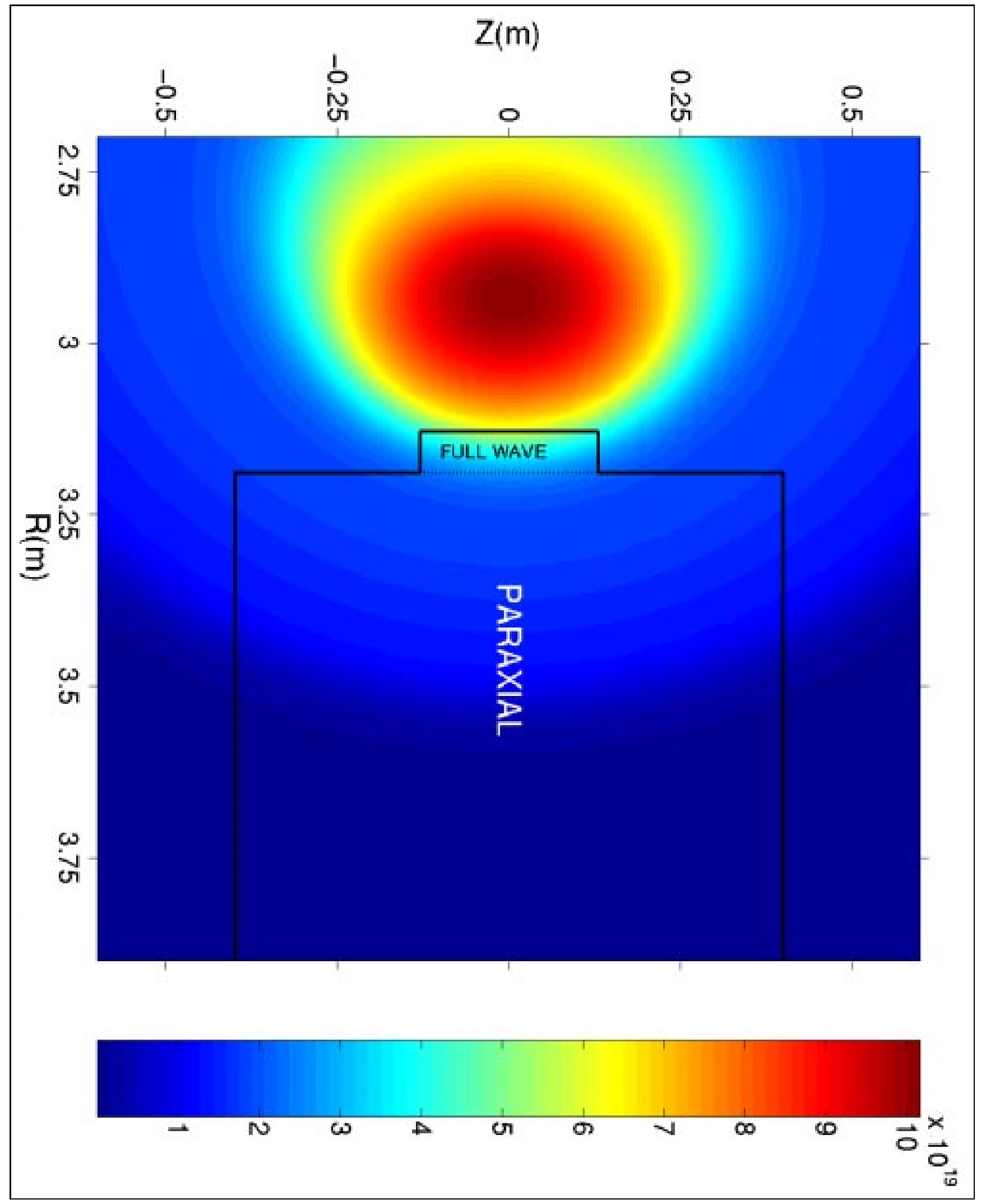




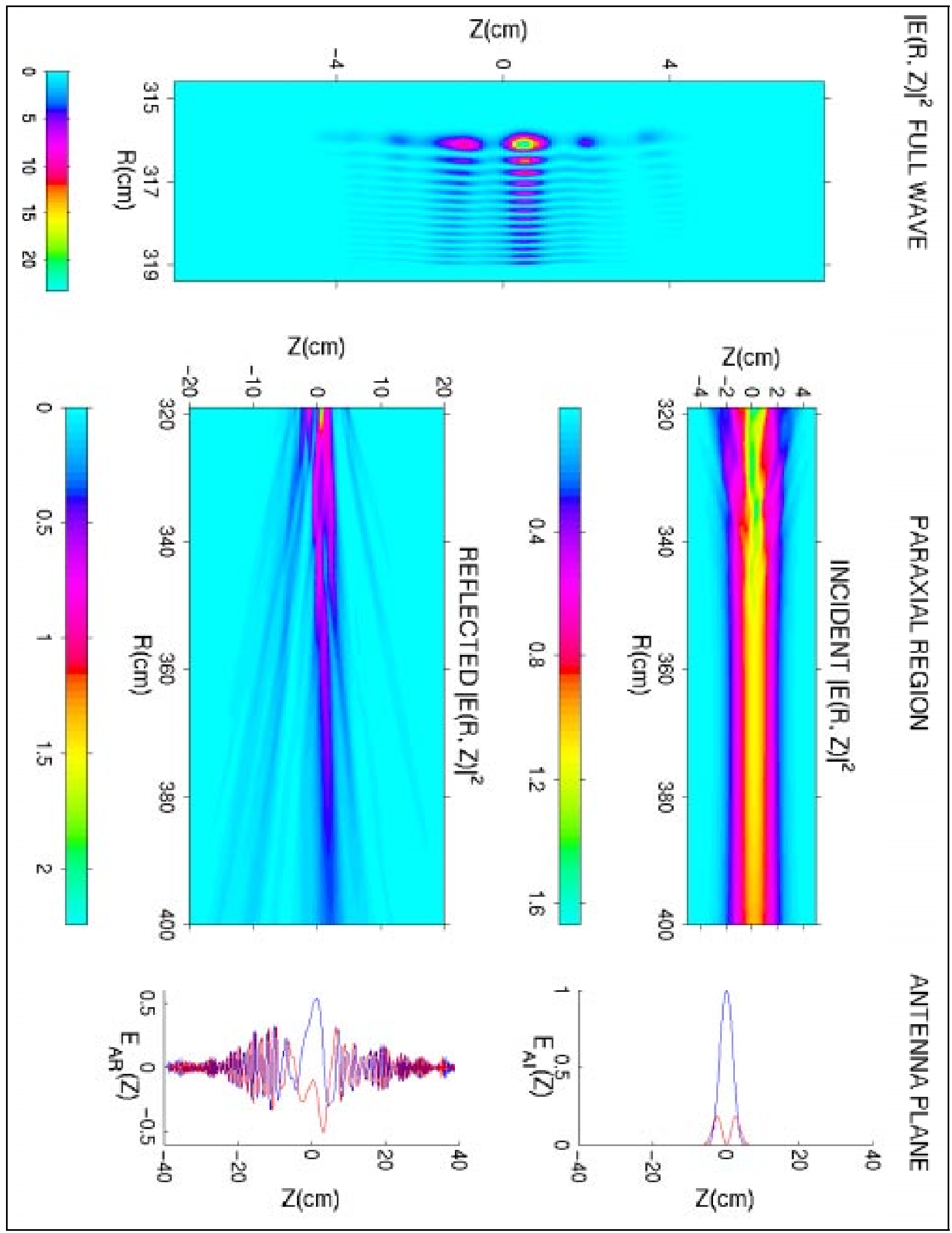




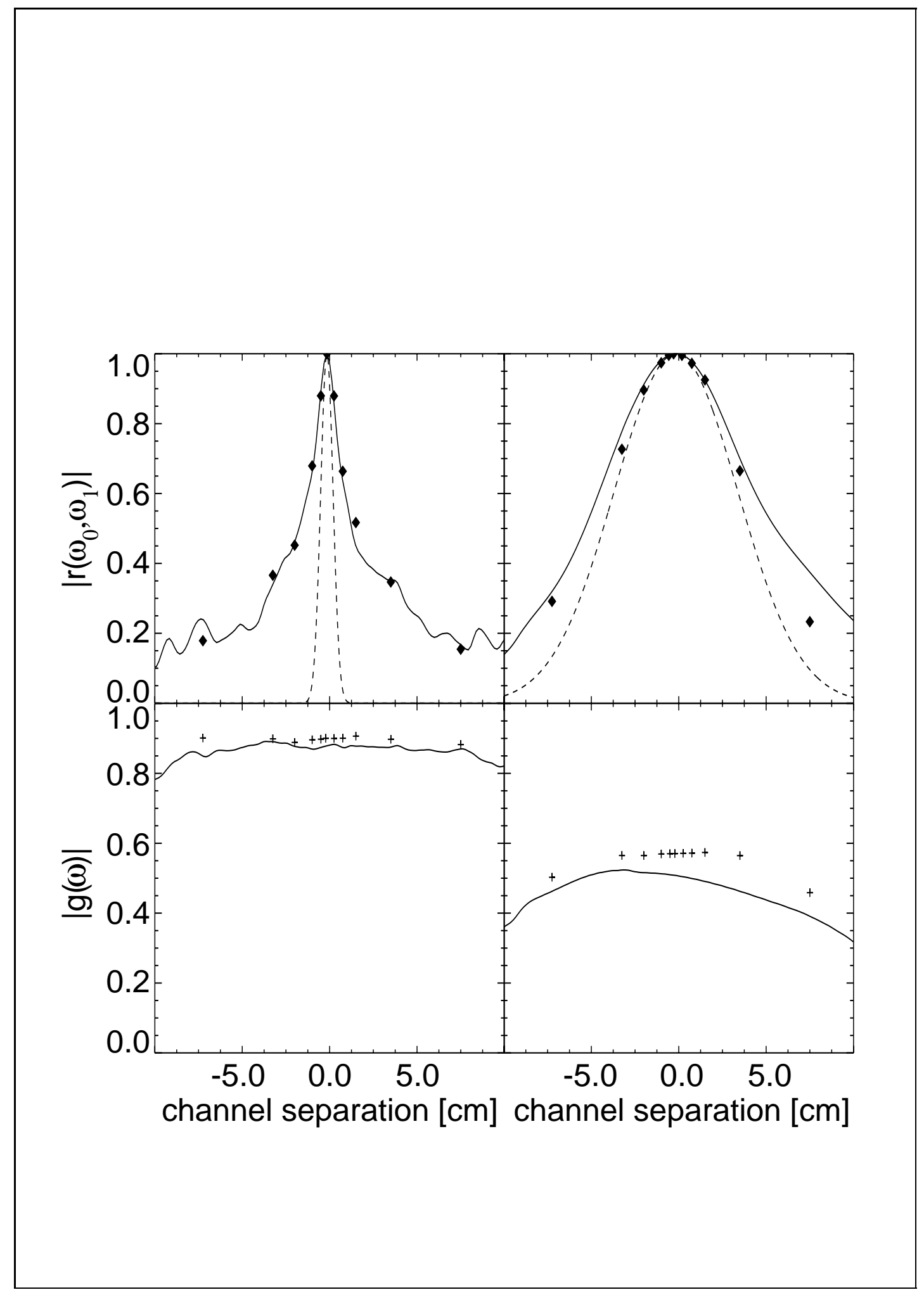




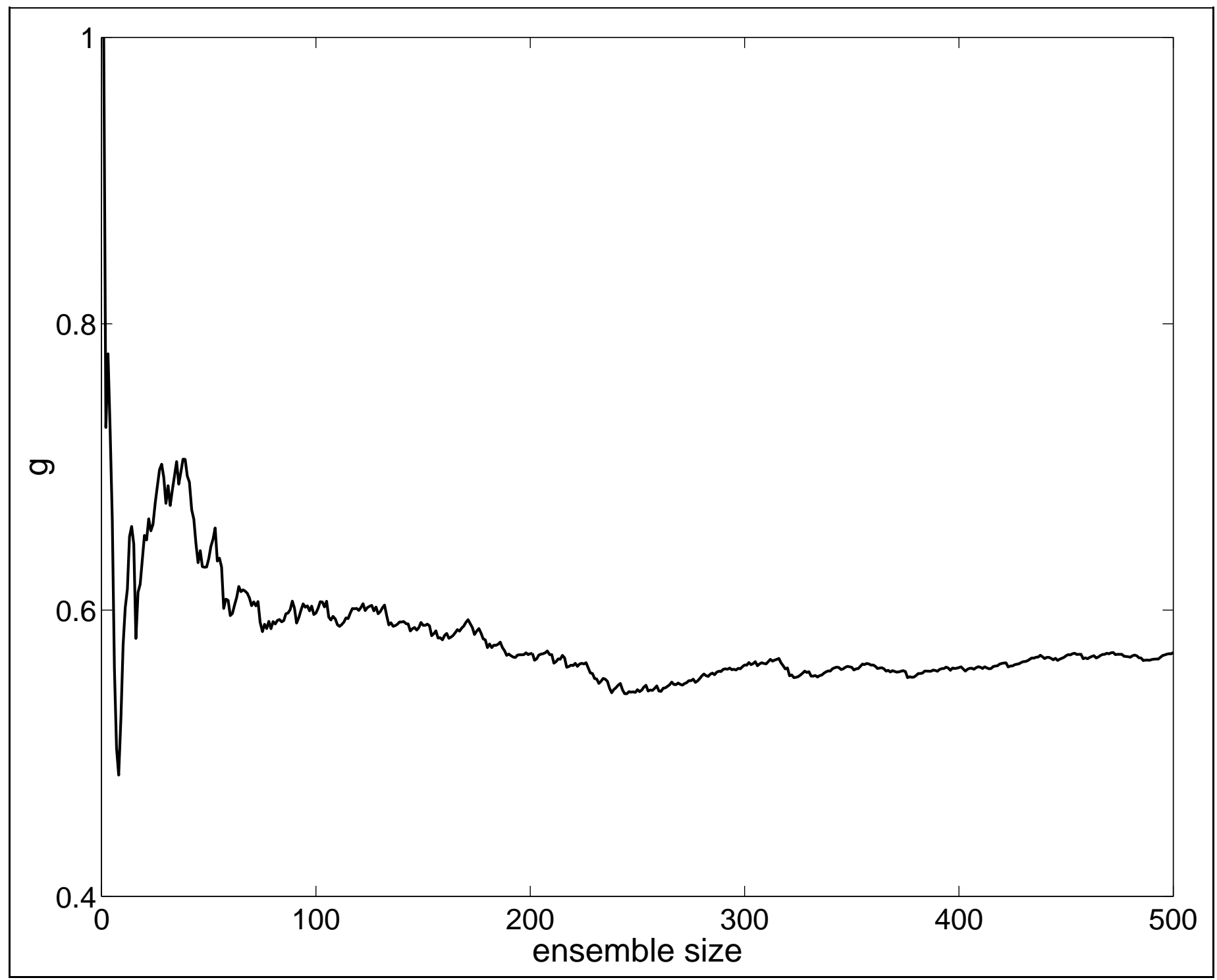




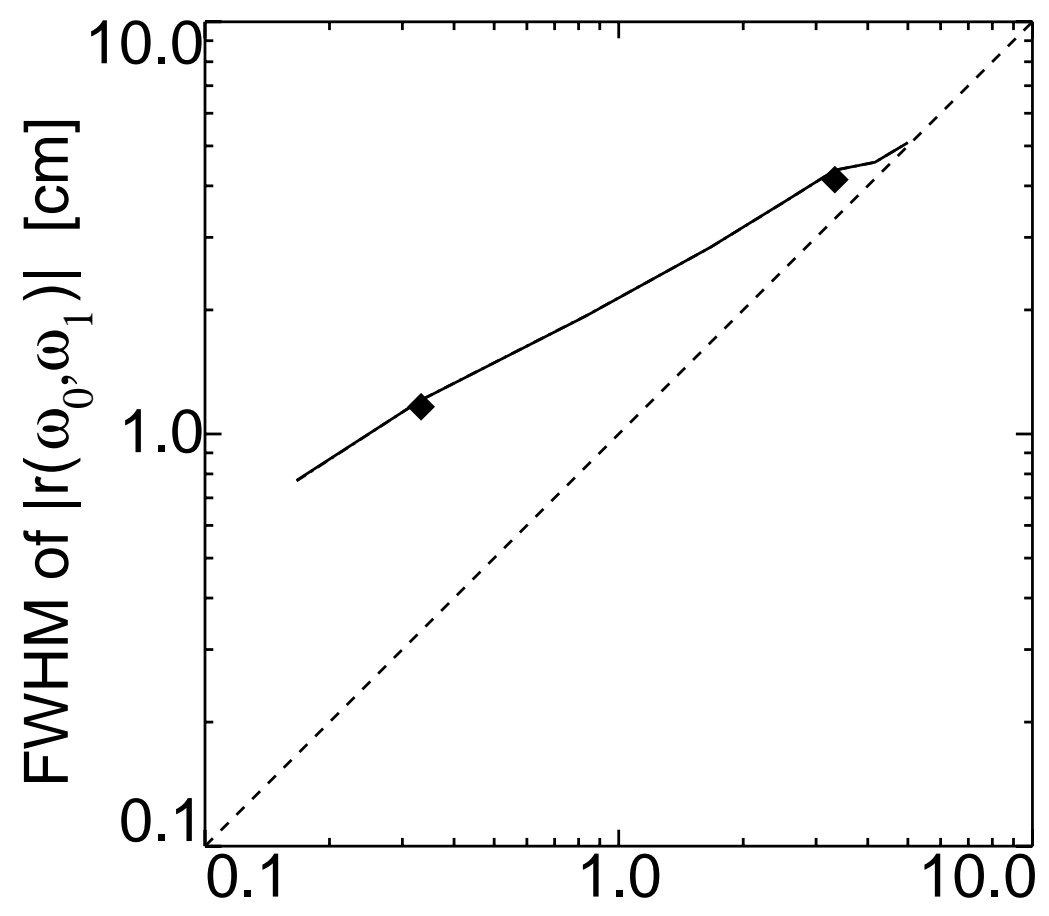

FWHM density correlation function [cm] 


\section{External Distribution}

Plasma Research Laboratory, Australian National University, Australia

Professor I.R. J ones, Flinders University, Australia

Professor J oão Canalle, Instituto de Fisica DEQ/IF - UERJ , Brazil

Mr. Gerson O. Ludwig, Instituto Nacional de Pesquisas, Brazil

Dr. P.H. Sakanaka, Instituto Fisica, Brazil

The Librarian, Culham Laboratory, England

Library, R61, Rutherford Appleton Laboratory, England

Mrs. S.A. Hutchinson, JET Library, England

Professor M.N. Bussac, Ecole Polytechnique, France

Librarian, Max-Planck-Institut für Plasmaphysik, Germany

J olan Moldvai, Reports Library, MTA KFKI-ATKI, Hungary

Dr. P. Kaw, Institute for Plasma Research, India

Ms. P.J . Pathak, Librarian, Insitute for Plasma Research, India

Ms. Clelia De Palo, Associazione EURATOM-ENEA, I taly

Dr. G. Grosso, Instituto di Fisica del Plasma, Italy

Librarian, Naka Fusion Research Establishment, J AERI, J apan

Library, Plasma Physics Laboratory, Kyoto University, J apan

Research Information Center, National Institute for Fusion Science, J apan

Dr. O. Mitarai, Kyushu Tokai University, J apan

Library, Academia Sinica, Institute of Plasma Physics, People's Republic of China

Shih-Tung Tsai, Institute of Physics, Chinese Academy of Sciences, People's Republic of China

Dr. S. Mirnov, TRINITI, Troitsk, Russian Federation, Russia

Dr. V.S. Strelkov, Kurchatov Institute, Russian Federation, Russia

Professor Peter Lukac, Katedra Fyziky Plazmy MFF UK, Mlynska dolina F-2, Komenskeho Univerzita, SK-842 15 Bratislava, Slovakia

Dr. G.S. Lee, Korea Basic Science Institute, South Korea

Mr. Dennis Bruggink, Fusion Library, University of Wisconsin, USA

Institute for Plasma Research, University of Maryland, USA

Librarian, Fusion Energy Division, Oak Ridge National Laboratory, USA

Librarian, Institute of Fusion Studies, University of Texas, USA

Librarian, Magnetic Fusion Program, Lawrence Livermore National Laboratory, USA

Library, General Atomics, USA

Plasma Physics Group, Fusion Energy Research Program, University of California at San Diego, USA

Plasma Physics Library, Columbia University, USA

Alkesh Punjabi, Center for Fusion Research and Training, Hampton University, USA

Dr. W.M. Stacey, Fusion Research Center, Georgia Institute of Technology, USA

Dr. J ohn Willis, U.S. Department of Energy, Office of Fusion Energy Sciences, USA

Mr. Paul H. Wright, Indianapolis, Indiana, USA 
The Princeton Plasma Physics Laboratory is operated by Princeton University under contract with the U.S. Department of Energy.

\author{
Information Services \\ Princeton Plasma Physics Laboratory \\ P.O. Box 451 \\ Princeton, NJ 08543
}

Phone: 609-243-2750

Fax: 609-243-2751

e-mail: pppl_info@pppl.gov

Internet Address: http://www.pppl.gov 